\title{
Late toxicities after intensity-modulated radiotherapy for nasopharyngeal carcinoma: patient and treatment-related risk factors
}

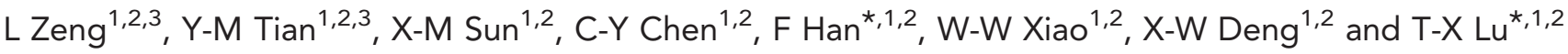 \\ ${ }^{1}$ State Key Laboratory Oncology in South China; Collaborative Innovation Center of Cancer Medicine, Guangzhou, People's \\ Republic of China and '2Department of Radiation Oncology, Sun Yat-Sen University Cancer Center, 651 Dong Feng Road East, \\ Guangzhou, People's Republic of China
}

Background: The objective of this study is to analyse the factors affecting late toxicity for nasopharyngeal carcinoma (NPC) patients treated with intensity-modulated radiotherapy (IMRT).

Methods: Seven hundred and eighty-nine consecutive NPC patients treated with IMRT at our centre from January 2003 to February 2008 were retrospectively analysed. Radiotherapy-related complications were categorised using the RTOG Late Radiation Morbidity Scoring Criteria and the Common Terminology Criteria for Adverse Events (Version 3.0). Two hundred and thirty-three patients were treated with IMRT alone (group 1) and 556 patients underwent cisplatin-based chemotherapy (group 2).

Results: Median follow-up was 65 months (range, 4-106 months). The 5-year major late toxicity rate was significantly greater in group 2 than group $1(63.2 \%$ vs $42.0 \%, P<0.001)$. Multivariate analyses showed that $\mathrm{N}$ category, $\mathrm{T}$ category and chemotherapy were significant factors. The maximal dose (Dmax) to the temporal lobe was a significant factor affecting temporal lobe injury (TLI), with a hazard ratio of 1.26 (95\% confidence interval $(\mathrm{Cl}), 1.18-1.35 ; \mathrm{P}<0.001)$ per 1 -Gy increase. The 5-year TLI rate increased from $0.8 \%$ for 284 lobes with Dmax $<65.77$ Gy to $27.1 \%$ for 176 lobes with greater doses $(P<0.001)$. Logistic regression showed that the hazard ratio attributed to the parotid gland mean dose was $1.36(95 \% \mathrm{Cl}, 1.21-1.53 ; P<0.001)$ per 1-Gy increase. Chemotherapy was not a significant factor $(P=0.211)$.

Conclusion: With the application of IMRT, the incidence of radiation-related complications has been reduced except for TLI. The significant factors affecting the risk of TLI included T category, chemotherapy and Dmax.

Primary radiotherapy (RT) has been the mainstay of treatment for patients with non-disseminated nasopharyngeal carcinoma (NPC), with an overall local control rate of $80-85 \%$ achieved through the use of the conventional Ho's two-dimensional (2D) planning $\mathrm{H}$ technique (Lee et al, 2005; Leung et al, 2005). Some studies have shown that a local boost with brachytherapy or the use of stereotactic RT after treatment with a $2 \mathrm{D}$ planning technique can significantly improve the local control rate to $>90 \%$ (Chen et al, 2006; Leung et al, 2008). However, this comes at the price of an increased risk of late toxicity. In addition, the failure rate is still high in patients with parapharyngeal involvement and stage T3-4 disease as a result of poor target coverage using the conventional 2D technique (Chau et al, 2001; Teo et al, 2004).

Intensity-modulated RT (IMRT) is a major breakthrough in the treatment of NPC. Compared with traditional 2D techniques, IMRT achieves good tumour coverage and normal organ sparing, allows dose escalation and achieves a high tumour control rate. Encouraging results with IMRT studies have been reported, and $>85 \%$ locoregional control has been consistently shown (Lee et al, 2002; Wolden et al, 2006; Tham et al, 2009; Wong et al, 2010;

${ }^{\star}$ Correspondence: Dr T-X Lu; E-mail: lutx@sysucc.org.cn or Dr F Han; E-mail: hanfei@sysucc.org.cn
${ }^{3}$ These authors contributed equally to this work.

Received 25 August 2013; revised 9 October 2013; accepted 21 October 2013; published online 19 November 2013

(c) 2014 Cancer Research UK. All rights reserved 0007 - 0920/14 
$\mathrm{Ng}$ et al, 2011). However, published literature reporting late morbidity has sometimes been selective or inconsistent (Lee et al, 2002; Wolden et al, 2006; Wong et al, 2010). Published studies have either involved a small size, have short- to medium-term follow-up or did not include detailed analyses of late RT-related complications. The accurate correlation of dose with late toxicity from patients with dose-volume histogram (DVH) data and long follow-up is grossly lacking.

Therefore, we initiated this study to estimate the relationship between the doses received by the organs at risk (OARs) at the nasopharyngeal region and the respective incidence of late toxicity and to assess the increase in risk incurred by chemotherapy.

\section{MATERIALS AND METHODS}

Patients characteristics. A total of 789 patients with newly diagnosed, non-metastatic and histologically confirmed NPC, treated with IMRT at our cancer centre from January 2003 to February 2008, were analysed. Of the 789 patients, 620 were men and 169 were women, with a men/women ratio of 3.7:1. The median age was 43 years (range, 13-78 years). Histologically, $7.2 \%$ (57 out of 789) of the patients had World Health Organization (WHO) type II disease, 92.6\% (731 out of 789) had WHO type III and only one had WHO type I. The median follow-up period was 65 months (range, 4-106 months).

All NPC cases were restaged according to the seventh edition of the AJCC staging system. Of the 789 patients, 243 (30.8\%) were of stage I-II and $546(69.2 \%)$ were of stage III-IVB.

Table 1 lists the patient characteristic and tumour factors.

\section{Treatment methods}

Radiotherapy. The primary tumour area and the upper-neck area above the caudal edge of the cricoids cartilage were treated with
IMRT. Target volumes were delineated according to our institutional treatment protocol, the International Commission on Radiation Units and Measurements Reports 50 and 62 (Zhao et al, 2006; Xiao et al, 2011; Su et al, 2012a). The lower neck and the supraclavicular fossae were treated with a single anterior split field by conventional RT. Planning target volumes (PTVs) for all gross tumour volumes and CTVs were generated automatically after delineation of tumour targets according to the immobilisation and localisation uncertainties. Inverse planning was performed on the Corvus System for all patients using Simultaneous Modulated Accelerated Radiation Therapy boost RT (Butler et al, 1999). The prescribe dose was 68 Gy to the PTV of the GTVnx, 60 Gy to the PTV of CTV1 (i.e., high-risk regions), $54 \mathrm{~Gy}$ to the PTV of CTV2 (i.e., low-risk regions) and 60-66 Gy to the PTV of the GTVnd for the positive cervical lymph nodes in 30 fractions. For the GTV and CTV, the target volumes that received $>95 \%$ of the prescribed dose was used to reflect the target coverage. The dose received by each critical organ was limited to tolerance according to the RTOG 0225 protocol (Radiation Therapy Oncology Group, 2008). In addition, the prescribed dose to the lower neck and the supraclavicular fossae by irradiation with the conventional RT technique was $50 \mathrm{~Gy}$ out of 25 fraction for prophylactic intent and $60-66$ Gy out of 30-33 fractions for therapeutic intent.

Chemotherapy. During the study period, our institutional guidelines recommended RT alone for stage I to IIA patients, concurrent chemoradiotherapy for stage IIB patients, and both neoadjuvant or adjuvant chemotherapy and concurrent chemotherapy for those in stage III to IVA-B defined by the sixth edition of the AJCC staging system for NPC. Neoadjuvant or adjuvant chemotherapy consisted of cisplatin $\left(80 \mathrm{mg} \mathrm{m}^{-2}\right)$ with 5 -fluorouracil $\left(800 \mathrm{mg} \mathrm{m}^{-2}\right)$ continuous infusion for 5 days or cisplatin $\left(80 \mathrm{mg} \mathrm{m}^{-2}\right)$ with paclitaxel $\left(175 \mathrm{mg} \mathrm{m}^{-2}\right)$ recycled every 3 weeks for three cycles. Concomitant chemotherapy consisted of cisplatin $\left(80 \mathrm{mg} \mathrm{m}^{-2}\right)$

\section{Table 1. Patient characteristics, staging}

Comparison between groups

\begin{tabular}{|c|c|c|c|c|}
\hline Characteristic & Whole series $n=789(\%)$ & Group 1 (RT alone) $n=233(\%)$ & Group $2(C R T) n=556(\%)$ & $P$-value \\
\hline Age (years) & & & & 0.012 \\
\hline $\begin{array}{l}\text { Median } \\
\text { Range }\end{array}$ & $\begin{array}{c}43 \\
13-78\end{array}$ & $\begin{array}{c}44 \\
18-78\end{array}$ & $\begin{array}{c}43 \\
13-75\end{array}$ & \\
\hline Gender & & & & 0.691 \\
\hline $\begin{array}{l}\text { Men } \\
\text { Women }\end{array}$ & $\begin{array}{l}620(78.6) \\
169(21.4)\end{array}$ & $\begin{array}{r}181(77.7) \\
52(22.3)\end{array}$ & $\begin{array}{l}439(79.0) \\
117(21.0)\end{array}$ & \\
\hline Stage & & & & $<0.001$ \\
\hline $\begin{array}{l}\text { I-II } \\
\| I-I V B\end{array}$ & $\begin{array}{l}243(30.8) \\
546(69.2)\end{array}$ & $\begin{array}{r}164(70.4) \\
69(29.6)\end{array}$ & $\begin{array}{r}79(14.2) \\
477(85.8)\end{array}$ & \\
\hline T category & & & & $<0.001$ \\
\hline $\begin{array}{l}\text { T1 } \\
\text { T2 } \\
\text { T3 } \\
\text { T4 }\end{array}$ & $\begin{array}{l}164(20.8) \\
163(20.7) \\
303(38.4) \\
159(20.1)\end{array}$ & $\begin{array}{c}114(48.9) \\
60(25.8) \\
51(21.9) \\
8(3.4)\end{array}$ & $\begin{array}{c}50(9.0) \\
103(18.5) \\
252(45.3) \\
151(27.2)\end{array}$ & \\
\hline $\mathrm{N}$ category & & & & $<0.001$ \\
\hline $\begin{array}{l}\text { N0 } \\
\text { N1 } \\
\text { N2 } \\
\text { N3 }\end{array}$ & $\begin{array}{c}172(21.8) \\
372(47.2) \\
184(23.3) \\
61(7.7)\end{array}$ & $\begin{array}{c}116(49.8) \\
90(38.6) \\
24(10.3) \\
3(1.3)\end{array}$ & $\begin{array}{r}56(10.1) \\
282(50.7) \\
160(28.8) \\
58(10.4)\end{array}$ & \\
\hline
\end{tabular}


given on weeks 1,4 and 7 of RT, or cisplatin $\left(30 \mathrm{mg} \mathrm{m}^{-2}\right)$ given weekly.

Overall, 233 patients were treated with RT alone and 556 patients received chemotherapy. Concomitant chemotherapy was given to 244 patients: 79 had stage II and 165 had stage III-IV disease. The combination of neoadjuvant and concomitant chemotherapy was delivered to 268 patients with stage III-IV disease. Concomitant and adjuvant chemotherapy was delivered to 44 patients with stage III-IV disease. Reasons for deviation from institutional guidelines include patients' refusal and organ dysfunction, suggesting intolerance to neoadjuvant or adjuvant chemotherapy despite stage III-IV disease.

Assessment and statistical analysis. Patients were followed at least every 3 months during the first 3 years and every 6 months thereafter until death. The late toxicity were graded according to the Radiation Therapy Oncology Group radiation morbidity scoring criteria and the Common Terminology Criteria for Adverse Events (Version 3.0).

The defining major toxicities included temporal lobe necrosis, cranial neuropathy, damage to the brain stem, spinal cord, optic chiasm, endocrine dysfunction, eyeball damage, trismus and xerostomia of grade 2 or greater.

All events were measured from the date of beginning primary RT. To assess the toxicities attributable solely to the primary treatment, the observations were censored at the beginning of repeat RT for patients who underwent repeat RT for local relapse. The comparison of groups according to patient and tumour characteristics was made using $\chi^{2}$-test and Mann-Whitney's $U$-test. The actuarial rates were calculated using the KaplanMeier method and the differences were compared with the log-rank test. A multivariate analysis of different risk factors was conducted using the Cox proportional hazard model. Furthermore, the relationship of radiation dose and chemotherapy with xerostomia was tested by logistic regression model. All statistical analyses were performed using Statistical Package for Social Sciences.

\section{RESULTS}

Survival and patterns of treatment failure. The 5-year overall survival, disease-free survival, local recurrence-free survival, nodal recurrence-free survival and distant metastasis-free survival rates were $84.5 \%, 78.2 \%, 92.9 \%, 97.1 \%$ and $84.9 \%$, respectively. A total of $74(9.4 \%)$ patients developed disease recurrence, 123 (15.6\%) developed distant metastases and 149 (18.9\%) died. The median time to recurrence was 31 (range, 6-95) months and to development of distant metastases was 17 (range, 3-96) months.

Overall incidence of late toxicities. All patients had a minimum follow-up of 3 months and were analysed for late RT complications. The cumulative incidence of late toxicities was listed in Table 2 .

Altogether, 440 patients $(55.8 \%)$ had one or more major late toxicities. The median latent period was 23 months after the beginning of IMRT. The overall actuarial rate at 5 years was significantly greater in patients treated with chemoradiotherapy compared with those treated with IMRT alone $(63.2 \%$ vs $42.0 \%$, $P<0.001$; Table 3).

The significant factors were listed in Table 4 on multivariate analyses with age ( $\geqslant 43 v s<43$ years), T category (T3-4 vs T1-2),

Table 2. Cumulative incidence of late toxicities among patients treated with RT alone and CRT

\begin{tabular}{|c|c|c|c|c|c|c|}
\hline & \multicolumn{2}{|c|}{ Total } & \multirow[b]{2}{*}{$\begin{array}{c}\text { Group } 1 \text { (RT alone) } \\
\text { No. (\%) }\end{array}$} & \multirow[b]{2}{*}{$\begin{array}{c}\text { Group } 2 \text { (CRT) } \\
\text { No. (\%) }\end{array}$} & \multirow[b]{2}{*}{$\begin{array}{c}\text { Severe grade } \\
\text { No. (\%) }\end{array}$} & \multirow[b]{2}{*}{$\begin{array}{c}\text { Median latent } \\
\text { interval (months) }\end{array}$} \\
\hline & No. & (\%) & & & & \\
\hline Total no. of patients & 789 & 100 & $233(100)$ & $556(100)$ & $-(-)$ & - \\
\hline \multicolumn{7}{|c|}{ Neurological complications } \\
\hline $\begin{array}{l}\text { Temporal lobe injury } \\
\text { Cranial nerve palsies } \\
\text { Lhermittes' syndrome } \\
\text { Brachial plexopathy }\end{array}$ & $\begin{array}{c}59 \\
15 \\
12 \\
0\end{array}$ & $\begin{array}{l}7.5 \\
1.9 \\
1.5 \\
0\end{array}$ & $\begin{array}{c}5(2.1) \\
3(1.3) \\
4(1.7) \\
0\end{array}$ & $\begin{array}{c}54(9.7) \\
12(2.2) \\
8(1.4) \\
0\end{array}$ & $\begin{aligned} & 1(0.1) \\
- & (-) \\
- & (-) \\
- & (-)\end{aligned}$ & $\begin{array}{r}34 \\
36 \\
5 \\
-\end{array}$ \\
\hline \multicolumn{7}{|c|}{ Non-neurological complications } \\
\hline $\begin{array}{l}\text { Tinnitus } \\
\text { Otorroea } \\
\text { Hearing loss } \\
\text { Eyeball damage } \\
\text { Trismus } \\
\text { Dysphagia } \\
\text { Subcutaneous fibrosis }\end{array}$ & $\begin{array}{c}155 \\
28 \\
336 \\
5 \\
17 \\
11 \\
331\end{array}$ & $\begin{array}{r}19.6 \\
3.5 \\
42.6 \\
0.6 \\
2.2 \\
1.4 \\
42.0\end{array}$ & $\begin{array}{c}44(18.9) \\
8(3.4) \\
79(33.9) \\
0 \\
1(0.4) \\
4(1.7) \\
80(34.3)\end{array}$ & $\begin{aligned} 111 & (20.0) \\
20 & (3.6) \\
257 & (46.2) \\
5 & (0.9) \\
16 & (2.9) \\
7 & (1.3) \\
251 & (45.1)\end{aligned}$ & $\begin{array}{l}-(-) \\
-(-) \\
16(2.0) \\
3(0.3) \\
-(-) \\
-(-) \\
1(0.1)\end{array}$ & $\begin{array}{l}12 \\
12 \\
11 \\
36 \\
10 \\
34 \\
24\end{array}$ \\
\hline \multicolumn{7}{|l|}{ Endocrinopathy } \\
\hline $\begin{array}{l}\text { Primary hypothyroidism } \\
\text { Hypopituitarism } \\
\text { Xerostomia }^{\text {a }} \\
\text { Osteo-radionecrosis } \\
\text { Second cancer within RT } \\
\text { field }\end{array}$ & $\begin{array}{c}9 \\
17 \\
605 \\
0 \\
3\end{array}$ & $\begin{array}{c}1.1 \\
2.2 \\
78.1(605 / 775) \\
0 \\
0.4\end{array}$ & $\begin{array}{c}2(0.9) \\
3(1.3) \\
126 / 229(55.0) \\
0 \\
2(0.9)\end{array}$ & $\begin{array}{c}7(1.3) \\
14(2.5) \\
479 / 546(87.7) \\
0 \\
1(0.2)\end{array}$ & $\begin{array}{l}-(-) \\
-(-) \\
5(0.8) \\
-(-) \\
3(100)\end{array}$ & $\begin{array}{l}60 \\
58 \\
- \\
- \\
77\end{array}$ \\
\hline
\end{tabular}


CRT (yes vs no) and $\mathrm{N}$ category (N2-3 vs N0-1) as covariates. $\mathrm{N}$ category was a strongly significant factor for major late toxicity (hazard ratio, 1.35; 95\% confidence interval (CI), 1.10-1.66; $P=0.004$ ). $\mathrm{T}$ category was also a significant factor (hazard ratio, $1.27 ; 95 \% \mathrm{CI}$, $1.03-1.57 ; P=0.029$ ). The third significant factor was CRT (hazard ratio, $1.42 ; 95 \% \mathrm{CI}, 1.10-1.83 ; P=0.007)$.

Patient and treatment-related risk factors to different structures. A total of 59 patients (7.5\%) have developed temporal lobe injury (TLI). Among them, $43(72.9 \%)$ have radiation injuries in unilateral temporal lobes, $15(25.4 \%)$ in bilateral temporal lobes and $1(1.7 \%)$ simultaneously in unilateral temporal lobe and brain stem. Of the 59 patients, 47 were asymptomatic but 1 patient (from group 2) had grade 4 severity. Three patients (3 out of $327,0.9 \%$ ) had developed TLI for T1-2 patients. The incidences of TLI is $12.1 \%$ (56 out of 462 ) in T3-4 patients. The 5-year actuarial rate of TLI was significantly higher in group 2 compared with group 1 (10.1\% vs $1.9 \%, P<0.001)$.

Of the 460 evaluable temporal lobes, 48 had TLI. Multivariate analyses showed that adjusted for age, gender and mean dose to the temporal lobe, the hazard ratio attributed to the maximal dose (Dmax) to the temporal lobe was 1.26 (95\% CI, 1.18-1.35; $P<0.001)$, T category was 3.31 (95\% CI, $1.10-1.9 .99 ; P=0.030)$ and that to chemotherapy was 2.58 (95\% CI, 1.09-6.10; $P=0.030$, Table 5). Receiver operating characteristic curve analysis was used to evaluate different cut-off points for Dmax to the temporal lobe. The cut-off points for Dmax was $65.77 \mathrm{~Gy}$ (sensitivity 95.8\%, specificity $68.4 \%$, AUC $=0.883$; as shown in Figure 1 ). The 5 -year TLI rate increased from $0.8 \%$ for 284 lobes with Dmax $<65.77$ Gy to $27.1 \%$ for 176 lobes with greater doses $(P<0.001)$.

A total of 15 patients (1.9\%) developed Lhermittes' syndrome. No severe radiation-induced myelitis was observed. The 5-year rate

\begin{tabular}{|c|c|c|c|}
\hline Toxicity & Group 1 (\%) & Group 2 (\%) & $\boldsymbol{P}$-value \\
\hline Temporal lobe injury & 1.9 & 10.1 & $<0.001$ \\
\hline Cranial neuropathy & 1.4 & 2.0 & 0.345 \\
\hline Lhermittes' syndrome & 1.3 & 1.5 & 0.819 \\
\hline Hypopituitarism & 1.5 & 1.9 & 0.162 \\
\hline Xerostomia (grade $\geqslant 2$ ) & $15.9^{a}$ & $20.1^{a}$ & 0.163 \\
\hline Hearing impairment & 33.6 & 46.7 & 0.001 \\
\hline Eyeball damage & 0 & 1.0 & 0.135 \\
\hline Trismus & 0.5 & 3.0 & 0.030 \\
\hline Any complication & 42.0 & 63.2 & $<0.001$ \\
\hline
\end{tabular}

of Lhermittes' syndrome increased only slightly from $1.3 \%$ in group 1 to $1.5 \%$ in group $2(P=0.819)$.

Fifteen patients (1.9\%) developed cranial neuropathy. The upper-group cranial nerves were affected in nine and the lowergroup cranial nerves in six patients. The 5 -year rate was similar between group 1 and group $2(1.4 \%$ vs $2.0 \%, P=0.345)$.

Seventeen patients (2.2\%) developed hypopituitary hormonal anomalies that required replacement medication. The 5-year rate increased from $1.5 \%$ in group 1 to $1.9 \%$ in group 2 ; the difference was insignificant $(P=0.162)$.

The most common radiation-related complication was xerostomia. Of 775 evaluable patients, a total of 605 patients (78.1\%) had xerostomia at 12 months after RT. The 1-year rate of grade 2 or greater was similar between group 1 and group 2 (15.9\% vs $20.1 \%, P=0.163)$. To ensure that xerostomia was induced primarily by the radiation treatment, patients with moderate to severe xerostomia at baseline were excluded from the analysis. The dosimetric data of 257 patients was collected. The parotid gland mean dose was $31.3 \mathrm{~Gy}$ (median:30.9, range, 25.3-49.5). The relationships between xerostomia $(G \geqslant 2)$ at 1 year after RT and patient age $(\geqslant 43$ vs $<43$ years), gender, $\mathrm{T}$ category, $\mathrm{N}$ category, chemotherapy (yes $v s$ no) and parotid gland mean dose were analysed using logistic regression model. Logistic regression showed that the hazard ratio attributed to the parotid gland mean dose was 1.36 (95\% CI, 1.21-1.53; $P<0.001)$ per 1 -Gy increase. Neither age $(P=0.289)$ nor chemotherapy $(P=0.211)$ was a significant factor. The 1 -year xerostomia of grade 2 or greater was significantly greater for those with parotid gland mean dose $\geqslant 30.9$ Gy than for those with lower doses (24.0\% vs $5.5 \%)$.

A total of 337 patients (42.7\%) developed hearing loss that persisted for over 90 days from the beginning of RT. The severity of the hearing loss was grade 3 in 13 patients (1.6\%) and grade 4 in 3 patients $(0.4 \%)$. The 5 -year rate was significantly greater in group 2 than in group $1(46.7 \%$ vs $34.1 \%, P=0.001)$. Multivariate analysis showed that chemotherapy and $\mathrm{T}$ category were significant factors (Table 4). The hazard ratio attributed to $\mathrm{T}$ category was 1.30 (95\% CI, 1.02-1.66, $P=0.038)$. The hazard ratio attributed to chemotherapy was 1.33 (95\% CI, 1.01-1.76, $P=0.042$ ).

Seventeen patients developed grade 1 trismus. There was no grade 2 to grade 4 trismus. The 5 -year rate increased from $0.5 \%$ in group 1 to $3.0 \%$ in group 2; the difference was significant $(P=0.030)$. However, multivariate analysis showed that age was a significant factor for trismus (Table 4). The hazard ratio was 3.85 (95\% CI, 1.10-13.48, $P=0.035)$. Chemotherapy was not a significant factor $(P=0.342)$.

Two patients developed a cataract and three had radiationinduced retinitis (they had exceptionally high doses to the lens and retina for extensive local infiltration into the orbits). Only two patients developed radiation-induced injury to brain stem. None of the patients developed bone necrosis or damage to the optic chiasm.

Table 4. Multivariate analysis of factors affecting late toxicities (patient-based for whole series)

\begin{tabular}{|l|c|c|c|c|}
\hline End point & Factor & $\boldsymbol{P}$-value & Hazard ratio (HR) & $\mathbf{9 5 \%}$ Cl for HR \\
\hline Any toxicity & N category (N2-3 vs N0-1) & 0.004 & 1.35 & $1.10-1.66$ \\
& T category (T3-4 vs T1-2) & 0.029 & 1.27 & $1.03-1.57$ \\
& Chemotherapy (yes vs no) & 0.007 & 1.42 & $1.10-1.83$ \\
\hline Hearing loss & T category (T3-4 vs T1-2) & 0.038 & 1.30 & $1.02-1.66$ \\
& Chemotherapy (yes vs no) & 0.042 & 3.58 & $1.01-1.76$ \\
\hline Trismus & Age ( $\geqslant 43$ vs <43 years) & 0.035 & 3.85 & $1.10-13.48$ \\
\hline \multicolumn{2}{|l|}{ Abbreviations: Cl= confidence interval; Dmax = maximal dose (as continuous variable); HR=hazard ratio. } \\
\hline
\end{tabular}




\begin{tabular}{|c|c|c|c|c|}
\hline \multicolumn{5}{|c|}{$\begin{array}{l}\text { Table } 5 \text {. Multivariate analysis on radiation dose anc } \\
\text { affecting late toxicities (organ-based for patients) }\end{array}$} \\
\hline End point & Factor & $\boldsymbol{P}$-value & HR & $\begin{array}{l}95 \% \mathrm{Cl} \\
\text { for } \mathrm{HR}\end{array}$ \\
\hline Temporal lobe injury & $\begin{array}{c}\text { Dmax (per Gy) } \\
\text { Chemotherapy (yes vs no) } \\
\text { T category (T3-4 vs T1-2) }\end{array}$ & $\begin{array}{r}<0.001 \\
0.030 \\
0.033\end{array}$ & $\begin{array}{l}1.26 \\
2.58 \\
3.31\end{array}$ & $\begin{array}{l}1.18-1.35 \\
1.09-6.10 \\
1.10-9.99\end{array}$ \\
\hline
\end{tabular}

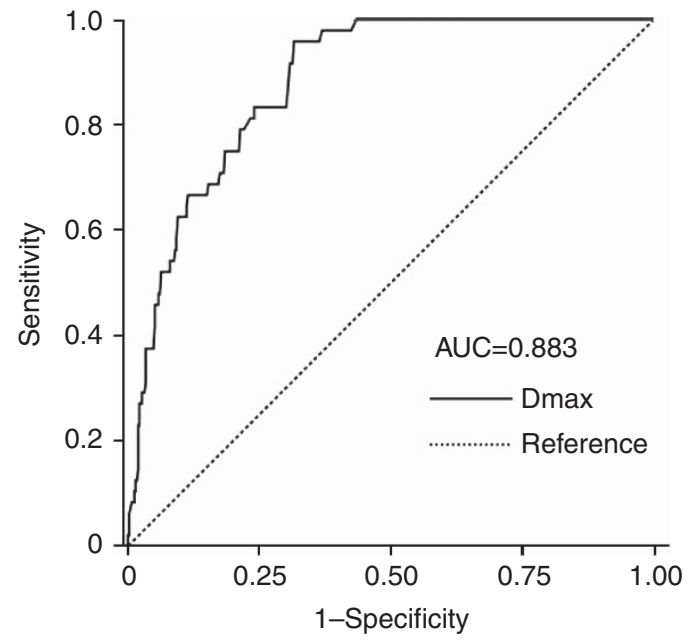

Figure 1. Receiver operator characteristic curves for diagnosis of the Dmax to temporal lobe for 460 evaluable temporal lobes.

\section{DISCUSSION}

The last decades have witnessed impressive advances in the radiotherapeutic management of NPC. Extensively used IMRT, magnetic resonance imaging and combined chemoradiotherapy have been reported to provide encouraging treatment outcome (Lee et al, 2002; Wolden et al, 2006; Tham et al, 2009; Wong et al, 2010; Ng et al, 2011). Hence, survivorship issues become crucial. Late toxicities can be life-threatening or significantly erode the patient's quality of life (QoL) and functional status. Assessing of QoL for NPC survivors has become an important issue in longterm patient care. However, published data of IMRT for late toxicities have sometimes been selective or inconsistent. The current study has provided an opportunity to assess not only the tolerance to IMRT alone but also the increase in risk incurred with the addition of chemotherapy. This is one of the largest series irradiated with IMRT, providing detailed DVH data on normal structures for assessing the dose relationship for various OARs.

The TLI is a serious late sequela after radical RT of NPC and adversely affects the patient's QoL and survival. Tian et al (2002) have reported the average incidence of radiation encephalopathy in NPC patients is $1.9 \%$ (range, $0.4-2.6 \%$ ) after 2D-RT and majority of the patients are treated with 2-Gy daily fractions for a total dose of nearly $70 \mathrm{~Gy}$. With IMRT, the incidence of TLI is expected to decrease. However, the incidence of $7.5 \%$ in this study is relatively higher compared with those in most previous reports. The present series has revealed that TLI is more likely to happen in patients with advanced disease, because their temporal lobes are more likely to be involved in the radiation fields, no matter using IMRT or 2D-RT techniques. However, IMRT offers better temporal lobe sparing in patients with early $\mathrm{T}$-stage disease compared with 2D-RT. In order to achieve satisfactory dose coverage to the targets in advanced T-stage NPC, a part of temporal lobes unavoidably receive high-dose irradiation due to proximity to the targets. In the present study, only three patients (3 out of $327,0.9 \%$ ) has developed TLI for T1-2 patients. In contrast, the incidences of TLI is $12.1 \%$ (56 out of 462 ) in T3-4 patients. Thus, for advanced T-stage NPC, IMRT seems to be failed to reduce TLI compared with 2D-RT. In addition, this may also be due to larger fractional dose in IMRT. Lawrence et al (2010) reported that the brain is especially sensitive to fraction sizes $>2$ Gy. However, at present, there is no consensus on the optimal dose and fractionation schemes in IMRT.

In the current series, the use of chemotherapy increased the incidence of TLI. Multivariate analysis showed that chemotherapy was an independent factor. The majority of TLI happened in patients with advanced $\mathrm{T}$ stage. Chemoradiotherapy is a standard treatment modality for advanced T-stage disease. Owing to the retrospective nature of this study, it was hard to know whether the reason for the fact that most TLI happened in patients with advanced $\mathrm{T}$ stage is because temporal lobes unavoidably received high-dose irradiation or because chemotherapy increased the incidence of TLI. Further research is needed to explore this issue.

This study has also shown that Dmax of temporal lobe is relevant to TLI. The 5-year TLI rate increased from $0.8 \%$ for 284 lobes with $\mathrm{Dmax}<65.77 \mathrm{~Gy}$ to $27.1 \%$ for 176 lobes with greater doses $(P<0.001)$. Su et al $(2012 \mathrm{~b})$ reported that TLI was not observed in patients with $\mathrm{Dmax}<64$ Gy or D1cc $<52 \mathrm{~Gy}$, but with the increase of Dmax and D1cc, that is, when Dmax $\geqslant 64$ Gy or $\mathrm{D} 1 \mathrm{cc} \geqslant 52 \mathrm{~Gy}$, the incidence of TLI increased significantly. This result concurred closely with our findings.

Although NPC can be treated effectively with non-IMRT planning technique, many patients complain of permanent xerostomia as a result of the delivery of a near full dose of radiotherapy to the bilateral parotid glands. The degree of xerostomia is largely dependent on the dose/volume of the salivary gland in the radiation field. The IMRT can limit the dose delivered to these glands without compromising tumour coverage. Many clinical studies have shown a lesser degree of xerostomia after IMRT relative to the $2 \mathrm{D}$ technique. Pow et al (2006) showed that IMRT was significantly better than conventional RT in terms of parotid sparing and improved QoL for early-stage NPC. Lee et al (2009) also showed that only $14 \%$ of patients reported grade 2 xerostomia at 1 year from the start of IMRT and that $35 \%$ of the patients had no complaints of xerostomia at all. The result from the present study concurred closely with that from Lee's study.

Hey et al (2009) reported on lower parotid gland tolerance doses for chemoradiotherapy versus RT alone. In contrast, Chao et al (2001) reported that induction or concomitant chemotherapy had no adverse role on whole mouth saliva flow rate at 6 months. Recently, Miah et al (2013) analysed the effect of concomitant chemotherapy on parotid gland function following head and neck IMRT, providing additional evidence that concomitant platinum chemotherapy had no significant adverse effect on subjective xerostomia symptoms and parotid saliva flow rates at 1 year after completing radiotherapy. In the current study, logistic regression showed that chemotherapy was not a significant factor affecting xerostomia. There was no difference in xerostomia $(G \geqslant 2)$ at 1 year after RT between IMRT alone and chemo-IMRT. This concurs with the results reported by Chao et al (2001) and Miah et al (2013).

The study reported by Lee et al (2009) showed that age, concurrent chemotherapy and the mean radiation dose to the cochlea were significant factors affecting deafness. In our study, advanced $\mathrm{T}$ stage and chemotherapy were negative prognostic factors for hearing impairment. The hearing impairment 
correlation with the dose to the cochlea had not been analysed, because there was no actual contouring of the cochlea and corresponding DVH data. The relationship of radiation dose to the cochlea with hearing impairment needs further research in future.

\section{CONCLUSION}

With the application of IMRT, the incidence of radiation-related complications has been reduced, except for TLI. Although IMRT optimises the radiation deposition in the tumour while sparing the adjacent normal structures, a high dose in the areas adjacent to normal temporal lobes seems to be unavoidable in some advanced T-stage NPC. Thus, for advanced T-stage NPC, IMRT fails to provide effective protection to TLs.

\section{CONFLICT OF INTEREST}

The authors declare no conflict of interest.

\section{REFERENCES}

Butler EB, Teh BS, Grant WH, Uhl BM, Kuppersmith RB, Chiu JK, Donovan DT, Woo SY (1999) Smart (simultaneous modulated accelerated radiation therapy) boost: a new accelerated fractionation schedule for the treatment of head and neck cancer with intensity modulated radiotherapy. Int J Radiat Oncol Biol Phys 45: 21-32.

Chao KS, Jo Deasy, Markman J, Haynie J, Perez CA, Purdy JA, Low DA (2001) A prospective study of salivary function sparing in patients with head-and-neck cancers receiving intensity-modulated or three-dimensional radiation therapy: initial results. Int J Radiat Oncol Biol Phys 49: 907-916.

Chau RM, Teo PML, Choi PH, Cheung KY, Lee WY (2001) Three-dimensional dosimetric evaluation of a conventional radiotherapy technique for treatment of nasopharyngeal carcinoma. Radiother Oncol 58: 143-153.

Chen HH, Tsai ST, Wang MS, Wu YH, Hsueh WT, Yang MW, Yeh IC, Lin JC (2006) Experience in fractionated stereotactic body radiation therapy boost for newly diagnosed nasopharyngeal carcinoma. Int J Radiat Oncol Biol Phys 66: 1408-1414.

Hey J, Setz J, Gerlach R, Vordermark D, Gernhardt CR, Kuhnt T (2009) Effect of cisplatin on parotid gland function in concomitant radiochemotherapy. Int J Radiat Oncol Biol Phys 75: 1475-1480.

Lawrence YR, Li XA, el Naqa I, Hahn CA, Marks LB, Merchant TE, Dicker AP (2010) Radiation dose-volume effects in the brain. Int J Radiat Oncol Biol Phys 76: S20-S27.

Lee AW, Ng WT, Hung WM, Choi CW, Tung R, Ling YH, Cheng PT, Yau TK, Chang AT, Leung SK, Lee MC, Bentzen SM (2009) Major late toxicities after conformal radiotherapy for nasopharyngeal carcinoma: patient and treatment-related risk factors. Int J Radiat Oncol Biol Phys 73: 1121-1128.

Lee AW, Sze WM, Au JSK, Leung SF, Leung TW, Chua DT, Zee BC, Law SC, Teo PM, Tung SY, Kwong DL, Lau WH (2005) Treatment results for nasopharyngeal carcinoma in the modern era: the Hong Kong experience. Int J Radiat Oncol Biol Phys 61: 1107-1116.

Lee N, Xia P, Quivey JM, Sultanem K, Poon I, Akazawa C, Akazawa P, Weinberg V, Fu KK (2002) Intensity-modulated radiotherapy in the treatment of nasopharyngeal carcinoma: an update of the UCSF experience. Int J Radiat Oncol Biol Phys 53: 12-22.

Lee N, Harris J, Garden AS, Straube W, Glisson B, Xia P, Bosch W, Morrison WH, Quivey J, Thorstad W, Jones C, Ang KK (2009) Intensitymodulated radiation therapy with or without chemotherapy for nasopharyngeal carcinoma: radiation therapy oncology group phase II trial 0225. J Clin Oncol 27: 3684-3690.

Leung TW, Tung SY, Sze WK, Wong FC, Yuen KK, Lui CM, Lo SH, Ng TY, O SK (2005) Treatment results of 1070 patients with nasopharyngeal carcinoma: An analysis of survival and failure patterns. Head Neck 27: $555-565$.

Leung TW, Wong VYW, Sze WK, Lui CM, Tung SY (2008) High-dose-rate intracavitary brachytherapy boost for early T stage nasopharyngeal carcinoma. Int J Radiat Oncol Biol Phys 70: 361-367.

Miah AB, Gulliford SL, Bhide SA, Zaidi SH, Newbold KL, Harrington KJ, Nutting CM (2013) The effect of concomitant chemotherapy on parotid gland function following head and neck IMRT. Radiother Oncol 106: 346-351.

Ng WT, Lee MC, Hung WM, Choi CW, Lee KC, Chan OS, Lee AW (2011) Clinical outcomes and patterns of failure after intensity-modulated radiotherapy for nasopharyngeal carcinoma. Int J Radiat Oncol Biol Phys 79: 420-428.

Pow EH, Kwong DL, McMillan AS, Wong MC, Sham JS, Leung LH, Leung WK (2006) Xerostomia and quality of life after intensity-modulated radiotherapy vs. conventional radiotherapy for early-stage nasopharyngeal carcinoma: Initial report on a randomized controlled clinical trial. Int J Radiat Oncol Biol Phys 66: 981-991.

Radiation Therapy Oncology Group (2008) Radiation Therapy Oncology Group Protocol 0225 http://www.rtog.org/members/protocols/0225/ 0225.pdf. Accessed: 26 August 2008.

Su SF, Han F, Zhao C, Chen CY, Xiao WW, Li JX, Lu TX (2012a) Long-term outcomes of early-stage nasopharyngeal carcinoma patients treated with intensity-modulated radiotherapy alone. Int J Radiat Oncol Biol Phys 82: 327-333.

Su SF, Huang Y, Xiao WW, Huang SM, Han F, Xie CM, Lu TX (2012b) Clinical and dosimetric characteristics of temporal lobe injury following intensity modulated radiotherapy of nasopharyngeal carcinoma. Radiother Oncol 104: 312-316.

Teo ML, Ma BY, Chan ATC (2004) Radiotherapy for nasopharyngeal carcinoma-transition from two-dimensional to three-dimensional methods. Radiother Oncol 73: 163-172.

Tham IW, Hee SW, Yeo RM, Salleh PB, Lee J, Tan TW, Fong KW, Chua ET, Wee JT (2009) Treatment of nasopharyngeal carcinoma using intensitymodulated radiotherapy- The National Cancer Centre Singapore experience. Int J Radiat Oncol Biol Phys 75: 1481-1486.

Tian Y, Gou R, Zhu MF (2002) Radiation encephalopathy in nasopharyngeal carcinoma patients in mainland China: a systematic evaluation. Chin J Oncol 24: 471-473.

Wolden SL, Chen WC, Pfister DG, Kraus DH, Berry SL, Zelefsky MJ (2006) Intensity-modulated radiation therapy (IMRT) for nasopharynx cancer: Update of the Memorial Sloan-Kettering experience. Int J Radiat Oncol Biol Phys 64: 57-62.

Wong FC, Ng AW, Lee VH, Lui CM, Yuen KK, Sze WK, Leung TW, Tung SY (2010) Whole-field simultaneous integrated-boost intensity-modulated radiotherapy for patients with nasopharyngeal carcinoma. Int J Radiat Oncol Biol Phys 76: 138-145.

Xiao WW, Huang SM, Han F, Wu SX, Lu LX, Lin CG, Deng XW, Lu TX, Cui NJ, Zhao C (2011) Local control, survival, and late toxicities of locally advanced nasopharyngeal carcinoma treated by simultaneous modulated accelerated radiotherapy combined with cisplatin concurrent chemotherapy: long-term results of a phase 2 study. Cancer 117: 1874-1883.

Zhao C, Lu TX, Han F, Lu LX, Huang SM, Deng XW, Lin CG, Cui NJ (2006) Clinical study of 139 nasopharyngeal carcinoma patients for intensity modulated radiation therapy. Chin J Radiat Oncol 15: 1-6.

This work is published under the standard license to publish agreement. After 12 months the work will become freely available and the license terms will switch to a Creative Commons AttributionNonCommercial-Share Alike 3.0 Unported License. 